\title{
Superconducting states in strongly correlated systems with nonstandard quasiparticles and real space pairing: an unconventional Fermi-liquid limit
}

\author{
J. Spałek $112 *$ \\ ${ }^{1}$ Marian Smoluchowski Institute of Physics, Jagiellonian University, Reymonta 4, PL-30-059 Kraków, Poland \\ 2 Faculty of Physics and Applied Computer Science, AGH University of Science and Technology, \\ Reymonta 19, PL-30-059 Kraków Poland
}

Received October 10, 2010, in final form October 14, 2010

\begin{abstract}
We use the concept of generalized (almost localized) Fermi Liquid composed of nonstandard quasiparticles with spin-dependence effective masses and the effective field induced by electron correlations. This Fermi liquid is obtained within the so-called statistically-consistent Gutzwiller approximation (SGA) proposed recently [cf. J. Jegdrak et al., arXiv: 1008.0021] and describes electronic states of the correlated quantum liquid. Particular emphasis is put on real space pairing driven by the electronic correlations, the Fulde-Ferrell state of the heavy-fermion liquid, and the d-wave superconducting state of high temperature cuprate superconductors in the overdoped limit. The appropriate phase diagrams are discussed showing in particular the limits of stability of the Bardeen-Cooper-Schrieffer (BCS) type of state.
\end{abstract}

Key words: correlated fermions, high temperature superconductivity, unconventional superconductivity, real space pairing, heavy fermions, $t-J$ model, Kondo pairing, FFLO state

PACS: $74.20 .-z, 71.27 .+a, 71.10 . C a$

\section{Introduction: Fermi liquids vs. correlated liquids}

The metals that we understand both qualitatively and (semi) quantitatively, are described by the Landau theory of Fermi liquids. It bases on the concept of the individual particle (electron and hole) excitations of electron gas, which survive in a liquid of interacting electrons. These excitations - quasiparticles - are long-lived, particulary near the Fermi surface, although they have renormalized ("dressed") characteristics such as the effective mass (but not charge) and related to it Fermi velocity. Such a metallic state is the point of departure of a very successful theoretical description of a wide class superconductors, the Bardeen-Copper-Schrieffer (BCS) theory of superconductivity. It relies on an attractive interaction created by the virtual exchange of (nonconserved) bosons-phonons, incorporated into the Fermi-liquid picture as an attractive interaction between the quasiparticles, and leads to an instability of the Fermi sea (as represented by states near the Fermi surface) against the bound-state (Cooper) pair formation, even for an arbitrarily small attraction magnitude. Such an instability, and resulting from it condensed metallic state of Cooper pairs in particular, was spectacularly embodied in the Landau Fermi-liquid picture by Bogoliubov and his coworkers [1] and others (the same method was presented independently by Valatin J.G. [2]; for its formulation in real-space form see [3] ) with the help of an anomalous averages expressing the superconducting order parameter within the mean-field (BCS) picture. The preservation of the Fermi-liquid picture even in the presence of pairing for ordinary metals was possible because the Cooper pairs are strictly speaking, not bosons, i.e. their fermionic origin persists, since the wave functions of individual pairs overlap strongly and therefore, their internal structure matters. The essence of the picture survives in the condensed state even when the coupling to the lattice is strong, if only the carrier concentration is large (i.e. the pair coherence length $\xi$ is still much

*E-mail: ufspalek@if.uj.edu.pl 
larger than the lattice parameter $a$ ). In that situation, the Coulomb repulsive interaction among electrons is additionally screened out effectively [4] and the condensed state persists, particulary for the three-dimensional metals and alloys, albeit with strongly renormalized transition temperature, with its maximal value of about $\mathrm{T} \simeq 30 \mathrm{~K}$.

The basic question concerning the BCS description validity arises when we consider strongly correlated systems, for which the single-particle energy or the Fermi energy, is comparable or even much smaller in magnitude than that of the Coulomb repulsion (represented then by the magnitude $U$ of the Hubbard intraatomic interaction). The situation is particulary acute for high temperature superconductors, since they can be regarded as quasi-two-dimensional metals in the normal state and the charge carrier concentration is maximally about $1 / 4-1 / 3$ electron per active (copper) site (formula unit), as is the case for e.g. $\mathrm{La}_{2-x} \mathrm{Sr}_{x} \mathrm{CuO}_{4}$ [5]. Under these circumstances, it seems questionable to start from the Landau Fermi-liquid theory, as the repulsive interaction encompasses all electrons, not only those near the Fermi surface. This situation is new also because the high-temperature superconducting state emerges by a relatively low hole doping $(x \gtrsim 0.05)$ from a generic antiferromagnetic 3d Mott-Hubbard insulator (for review see e.g.: [6]). We call the resultant metallic state, particulary close to the metal-insulator boundary, a correlated (nonFermi) quantum liquid. Nonetheless, the experiments show clearly, that as we increase the hole concentration $(x \gtrsim 0.15)$, the correlated-liquid state transforms gradually into the Fermi-liquid-like state. So minimally, when approaching the unconventional superconductivity of high-temperature or heavy-fermion systems far from the quantum critical point, we should be able to describe it via a some kind of a nonstandard Fermi-liquid, both in the normal and in the condensed superconducting phases. However, even in the at regime a novel unique feature or appears for high- $T_{\mathrm{c}}$ systems namely, when the carrier concentration increases and the systems reaches a metallic concentration, (albeit on the low side, $x \sim 1 / 3$ ), the superconductivity disappears altogether. The situation is illustrated in figure 1 . The phenomenon is universal to all the cuprates and related systems [5].

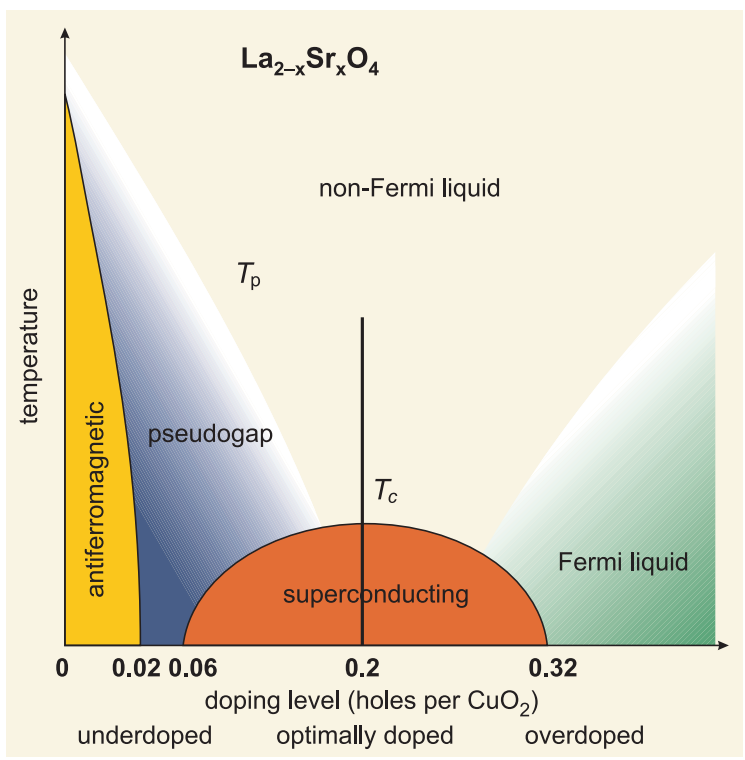

Figure 1. Schematic phase diagram for $\mathrm{La}_{2-x} \mathrm{Sr}_{x} \mathrm{CuO}_{4}$ and the division into three doping regimes specified. The vertical line defines roughly the optimal doping. The unconventional Fermi-liquid state roughly corresponds to the regime to the right of the vertical line, where the pseudogap onset temperature disappears.

This feature seems to rule out the usual pairing in reciprocal space and mediated solely by the electron-phonon lattice, since then with the growing carrier concentration the Coulomb repulsion is screened more effectively and the transition temperature should certainly not decrease. In this context, let us mention right away a natural concept of real space pairing induced by short range 
exchange interactions, the so-called t-J or related models 7 -10] (for recent review see: 11]), in which the pairing is caused by the neighboring spin-singlet correlations which in turn, are diluted upon the increasing the hole doping and eventually the paired state disappears above the upper critical concentration $n_{\mathrm{c}} \sim 1 / 4-1 / 3$, which represents some kind of critical concentration for local pair-hole dissociation.

The purpose of this chapter is to review our [12 15] and related (for review see [16]) works on real space paired states in the nonstandard Fermi-liquid regime. We discuss briefly both the heavyfermion and the high- $T_{\mathrm{c}}$ situations. But first, we overview briefly the relevant pairing mechanisms induced by the exchange interactions an their direct relation to onset of the antiferromagnetic insulating (Mott) state.

\section{Antiferromagnetic exchange interactions as such and as a source of real space pairing: a perspective}

\subsection{Kinetic-exchange and real space pairing: narrow band}

The relevant for our purpose history of antiferromagnetic exchange interactions in strongly correlated and insulating magnetic oxides bears its origin from the pioneering work of Anderson [17] who introduced the idea of kinetic exchange between the neighboring atomic $3 \mathrm{~d}$ states of transition-metal cations, each of which has an admixture of $2 \mathrm{p}$ states of surrounding $\mathrm{O}^{2-}$ anions. In this manner, the superexchange interaction via the nonmagnetic anions is expressed directly by that between the magnetic 3d cations. The Anderson's idea of kinetic exchange, applicable to the Mott insulators, has been applied subsequently also to the charge-transfer insulators [18] (see below).

The idea of kinetic exchange was extended subsequently to strongly correlated metals by Spałek, Oleś, and Chao [19]. Its essence can be summarized as follows [20]. In the simplest situation, we start from the Hubbard model representing a single narrow band and expressed in the Wannier representation, which has the form

$$
H=\sum_{i j \sigma} t_{i j} a_{i \sigma}^{\dagger} a_{j \sigma}+U \sum_{i} n_{i \uparrow} n_{i \downarrow}
$$

where the summation means that we take only the sites $i \neq j$ in the hopping part with the hopping integral $t_{i j}$ between them. The hopping range is determined by the number of coordination spheres, for which $t_{i j} \neq 0$. The second term represents the intraatomic Coulomb interaction only between the two particles with opposite spins $(\sigma=\uparrow$ and $\sigma=\downarrow)$. Here $i$ denotes symbolically the Wannier state $\left|W_{i}(\underline{\mathrm{r}})\right\rangle=\left|W\left(\underline{\mathrm{r}}-\underline{\mathrm{R}}_{i}\right)\right\rangle$ centered on site $i$. In the strong-correlation limit, we have $\left|t_{i j}\right| \ll U$ and hence the kinetic (bare band) energy, as represented by the first term, is much smaller than $U$ and thus can be regarded as a perturbation. In effect, the Hubbard Hamiltonian may be transformed in the first nontrivial (second) order in $t_{i j} / U$ to the following form [19, 20]

$$
\mathcal{H}_{t J}=\sum_{i j \sigma} t_{i j} b_{i \sigma}^{\dagger} b_{j \sigma}+\sum_{i j} J_{i j}\left(\mathbf{S}_{i} \cdot \mathbf{S}_{j}-\frac{1}{4} \nu_{i} \nu_{j}\right)+\sum_{i j k} \frac{t_{i j} t_{j k}}{U}\left(b_{i \sigma}^{\dagger} S_{j}^{\bar{\sigma}} b_{k \bar{\sigma}}-b_{i \sigma}^{\dagger} \nu_{j \bar{\sigma}} b_{k \sigma}\right) .
$$

The processes representing the dynamical processes are sketched in figure 2 . In the above expression $b_{i \sigma}^{\dagger} \equiv a_{i \sigma}\left(1-n_{i \bar{\sigma}}\right), \nu_{i \sigma} \equiv n_{i \sigma}\left(1-n_{i \bar{\sigma}}\right), \nu_{i} \equiv \sum_{\sigma} \nu_{i \sigma}$, and $S_{i}^{\sigma} \equiv b_{i \sigma}^{\dagger} b_{i \bar{\sigma}}$, with $\bar{\sigma} \equiv-\sigma$. The kineticexchange integral is $J_{i j}=2 t_{i j}^{2} / U$. In this Hamilltonian, we project out the local (site) double occupancies, as well as include both the two-site (virtual-exchange) and the three-site hopping terms. In the Mott-Hubbard insulating limit we have the conservation of particle number on each site, i.e. $n_{i \sigma}+n_{i \bar{\sigma}}=1$, and hence the effective Hamiltonian reduces to that of Anderson [16] in the single-band situation ( $\operatorname{spin} S=1 / 2$ ). One has also to note that the intersite Coulomb interaction $\sim \sum_{i j} K_{i j} n_{i} n_{j}$ has been neglected in (11). This term can be included in the analysis [21]. In general, the first term describes a nontrivial form of single-particle hopping, with the particles avoiding each other (no double occupancies), while the third term represents the nontrivial three-site hopping 


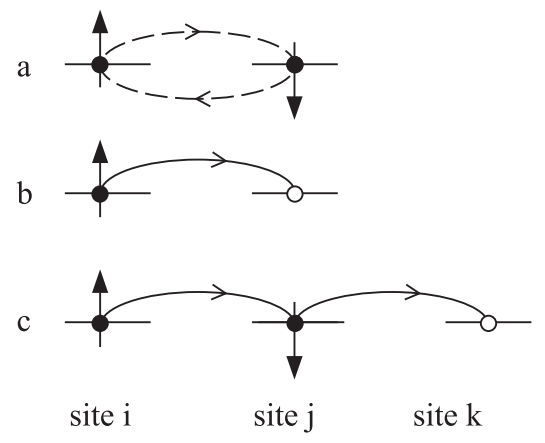

Figure 2. Three possible hopping processes determining the dynamics of Hamiltonian (2) in the lower Hubbard subband (i.e. for the band filling $n \leqslant 1$ ): (a) virtual hopping between single occupied neighboring sites that leads to their antiferromagnetic (kinetic) exchange interaction; (b) two-site single-particle hopping between the single occupied and the empty sites; and (c) three-site hopping between the singly occupied and the empty site via on intermediate single occupied site with opposite spin.

$k \rightarrow i$, with and without spin flip on the intermediate site $j$, respectively. In effect, one can say that in the metallic state (with the band filling $n=\left\langle n_{i}\right\rangle \neq 1$ ) we have $n N$ moving spins (or equivalently, of $N-N n=N(1-n)$ holes among the spins of the $N$-site system) that are correlated via antiferromagnetic interaction with its range equal to the number of coordination spheres we take, for which $t_{i j} \neq 0$. The state describing such liquid of moving spins and holes in between them is customarily called the resonating valence bond (RVB) state [10] although, strictly speaking, in the present situation the various pair spin-singlet configurations, taken into account in the RVB theory of Mott insulator [7-10], are intermixed with the hopping processes.

The effective Hamiltonian (2) contains essentially the intertwinned hopping (kinetic motion) and the spin correlations (kinetic exchange and 3-side hopping), as any of the terms do not commute with each other. So quite few nontrivial types the behavior may appear, since minimally the band and the spin-ordering effects compete viciously, as they become comparable near the Mott insulating limit [20]. Therefore, it was totally surprising at first (at least for the author), that the Hamiltonian (2) contains also an essentially new physics connected with the real space pairing [7-10], which effectively can be reduced to some form of BCS theory. A systematic approach to the concept of new pairing starts with defining the local pairing operators as [8]

$$
\left\{\begin{array}{l}
B_{i j}^{\dagger} \equiv \frac{1}{\sqrt{2}}\left(b_{i \uparrow}^{\dagger} b_{i \downarrow}^{\dagger}-b_{i \downarrow}^{\dagger} b_{i \uparrow}^{\dagger},\right), \\
B_{i j} \equiv \frac{1}{\sqrt{2}}\left(b_{i \downarrow} b_{i \uparrow}-b_{i \uparrow} b_{i \downarrow}\right)=\left(B_{i j}^{\dagger}\right)^{\dagger} .
\end{array}\right.
$$

In effect, one can rewrite the kinetic-exchange energy part in the form:

$$
\mathbf{S}_{i} \cdot \mathbf{S}_{j}-\frac{1}{4} \nu_{i} \nu_{j} \equiv-B_{i j}^{\dagger} B_{i j}
$$

What is even more surprising, the Hamiltonian (2) can be rewritten in the following closed form [8]:

$$
\mathcal{H}_{t J}=\sum_{i j \sigma} t_{i j} b_{i \sigma}^{\dagger} b_{j \sigma}-\sum_{i j k} \frac{2 t_{i j} t_{j k}}{U} B_{i j}^{\dagger} B_{k j} .
$$

In this form we see that the effective Hamiltonian contains in a direct manner an individual spin hopping (the first term) and the local pair binding (the $i=k$ part), as well as the 3 -site hopping for $i \neq k$. The beauty (and complication) of the problem is that the two terms can become comparable right at the lower (lower critical) concentration for the transition from an insulating antiferromagnetic to the superconducting state. This is the reason why superconductivity and antiferromagnetism can become intimately connected in the t-J model if only $B_{i j}$ are the forerunners 
of a condensed spin-singlet superconducting state. Parenthetically, because of the exclusion of the double-occupied site configurations $\left(B_{i i}^{\dagger}=B_{i i} \equiv 0\right)$, the pairing will have a nontrivial momentum $\hbar \mathbf{k}$ dependence of the superconducting gap. The superconducting state emerging out of this representation of the kinetic-exchange part is discussed in the next section. We should mention that the ideas of real-space pairing have been extended also to the case with orbital degeneracy in 22 .

\subsection{Kondo-interaction induced real space pairing: hybrizided-band case}

The Anderson-lattice model represents the simplest two-band situation, in which bare (starting) atomic strongly correlated states (of $3 \mathrm{~d}$ or $4 \mathrm{f}$ type, for example) are intermixed with starting atomic conduction ( $4 \mathrm{~s}$ or $\mathrm{d} 5-6 \mathrm{~s}$ type, respectively), which form already a band in the solid-state case even so the $3 \mathrm{~d}$ or $4 \mathrm{f}$ states remain atomic. This is easy to understand, since the higher atomic states as more extended collectivized first. The specific feature of the Anderson-lattice model is that the outermost valence states looses their atomic parity $\left((-1)^{l}\right.$ rule) due to their band character so it is possible to have (e.g. for $4 \mathrm{f}$ and the $5 \mathrm{~d}-6$ s interatomic mixing between the atomic (4f) and the Wannier 5d and 6s) states. This last circumstance is constitutes the physical origin of the model. Its formal structure from the first-quantization point of view is not so simple. However, in the second-quantization language it is easy to formulate the microscopic Hamiltonian, as the qualitative argument provided above is contained in the microscopic parameters. In effect, the starting Hamiltonian in the Wannier representation has the form

$\mathcal{H}=\sum_{m n \sigma} t_{m n} c_{n \sigma}^{\dagger} c_{n \sigma}+\epsilon_{\mathrm{f}} \sum_{i \sigma} N_{i \uparrow}+U \sum_{i \downarrow}+\sum_{i m \sigma} V_{i n}\left(f_{i \sigma}^{\dagger} c_{m \sigma}+c_{m \sigma}^{\dagger} f_{i \sigma}\right)-\mu\left(\sum_{i \sigma} N_{i \sigma}+\sum_{m \sigma} n_{m \sigma}\right)$,

where $i$ labels correlated atomic $(\mathrm{f}=3 \mathrm{~d}$ or $4 \mathrm{f})$ states, $(\mathrm{m}, \mathrm{n})$ label delocalized $c$ states, $N_{i \sigma} \equiv f_{i \sigma}^{\dagger} f_{i \sigma}$, and $V_{i m}$ represents the hybridization integral. In the limit $\left|V_{i m}\right| \ll\left|\epsilon_{\mathrm{f}}\right|$ and $U$, the above periodic Anderson Hamiltonian transforms into the Kondo Hamiltonian with the antiferromagnetic (kineticexchange) Kondo coupling between the itinerant and the localized electrons [23]. In the limit, the numbers of correlated and of the itinerant electrons are conserved separately. So this limit of the Anderson-lattice Hamiltonian, the so-called Kondo-lattice limit does not concern us here.

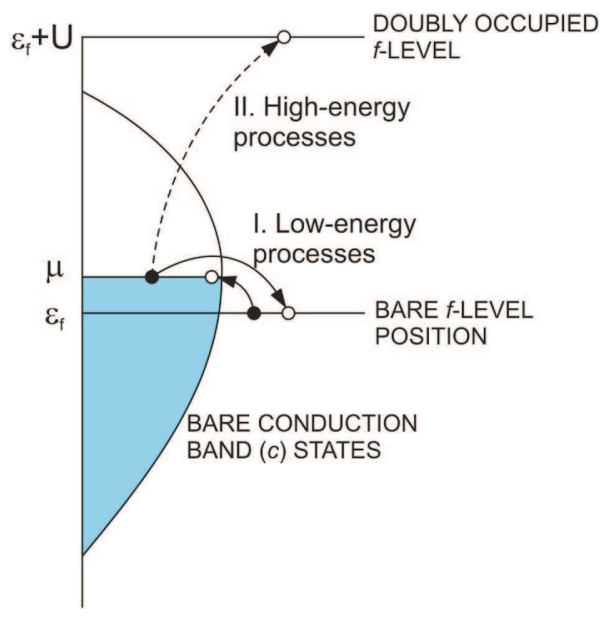

Figure 3. Schematic representation of hybridization-induced process as f-level occupied dependent hopping process and their division into low- and high-energy processes. The former (I) correspond to residual hybridization term in (8); the other (II) lead to the Kondo-type coupling which in turn is expressed as real-space hybrid pairing in (11) in the second order in $V /\left(\epsilon_{\mathrm{f}}\right)$.

The situation of interest for us here is provided by the condition that $\left|V_{\text {in }}\right| \ll U$, but $\left|V_{\text {in }}\right| \lesssim\left|\epsilon_{\mathrm{f}}\right|$, as represented schematically in figure 3. This means that we transform out only the high-energy terms of the order $V_{i m} / U$ and replace them with the effective kinetic-exchange interactions of the 
Kondo type, but leave the residual hybridization term $\sim V_{i m}\left(1-N_{\bar{\sigma}}\right) f_{i m}^{\dagger} c_{m}+\cdots$, untouched. In result the effective Hamiltonian in the second order acquires the approximate form [24]

$$
\begin{aligned}
\mathcal{H}= & \sum_{m n \sigma} t_{m n} c_{n \sigma}^{\dagger} c_{n \sigma}+\epsilon_{\mathrm{f}} \sum_{i \sigma} \nu_{i \sigma}+\sum_{i m \sigma} V_{i n}\left(1-N_{i \bar{\sigma}}\right)\left(f_{i \sigma}^{\dagger} c_{m \sigma}+c_{m \sigma}^{\dagger} f_{i \sigma}\right) \\
& +\sum_{i m} \frac{2 V_{i m}^{2}}{\epsilon_{\mathrm{f}}+U}\left(\mathbf{S}_{i} \cdot \mathbf{s}_{m}-\frac{1}{4} \nu_{i} n_{m}\right)+\frac{1}{\epsilon_{\mathrm{f}}+U} \sum_{i m n \sigma} V_{m i} V_{i n} S_{i}^{\sigma} c_{m \sigma}^{\dagger} c_{n \sigma}
\end{aligned}
$$

where now $t_{m n} \equiv t_{m n}-\mu \delta_{m n}$, and $\epsilon_{\mathrm{f}} \equiv \epsilon_{\mathrm{f}}-\mu$. As in the single-narrow-band case, we have projected out completely the double occupancies on a-site, as well as have defined the quantities $\nu_{i \sigma} \equiv N_{i \sigma}\left(1-N_{i \bar{\sigma}}\right), \nu_{i}=\sum_{\sigma} \nu_{i \sigma}, \mathbf{S}_{i} \equiv\left(S_{i}^{\sigma}, S_{i}^{z}\right) \equiv\left[f_{i \sigma}^{\dagger} f_{i \sigma}, \frac{1}{2}\left(\nu_{i \uparrow}-\nu_{i \downarrow}\right)\right]$ and $\mathbf{s}_{m}=\left(s_{m}^{\sigma}, s_{m}^{z}\right)=$ $\left[c_{m \sigma}^{\dagger} c_{m \bar{\sigma}},(1 / 2)\left(n_{m \uparrow}-n_{m \downarrow}\right) \sigma\right]$. The third and the fourth terms represent the truncated a-c mixing and the Kondo coupling, respectively. The Kondo antiferromagnetic exchange integral is $J_{i m}^{(K)}=$ $2 V_{i m}^{2} /\left(\epsilon_{\mathrm{f}}+U\right)$. The dynamical process leading to the above Hamiltonian have been sketched in figure 3 .

In analogy to the section 2.1 we introduce here the so-called hybrid pairing operators in real space

$$
\left\{\begin{array}{l}
A_{i m}^{\dagger}=\frac{1}{\sqrt{2}}\left[f_{i \uparrow}^{\dagger} c_{m \downarrow}^{\dagger}\left(1-N_{i \downarrow}\right)-f_{i \downarrow}^{\dagger} c_{m \uparrow}^{\dagger}\left(1-N_{i \uparrow}\right)\right], \\
A_{i m}=\left(A_{i m}^{\dagger}\right)^{\dagger}
\end{array}\right.
$$

In that situation, the hole affective Hamiltonian can be cast in a bit more compact form as follows

$$
\mathcal{H}=\sum_{m n \sigma} t_{m n} c_{m \sigma}^{\dagger} c_{n \sigma}+\sum_{i m \sigma}\left[V_{i n}\left(1-N_{i \bar{\sigma}}\right) f_{i \sigma}^{\dagger} c_{m \sigma}+H . c .\right]-\sum_{i m n} \frac{V_{i m} V_{i n}^{*}}{\epsilon_{\mathrm{f}}+U} A_{i m}^{\dagger} A_{i n} .
$$

In this form we have taken into account the fact, that the hybridization matrix element $V_{\text {in }}$ can be complex. Also, we can also include the higher order terms. In the fourth order the pairing part between f-electrons taken the form (5). The basic question when the Hamiltonian for e.g. magnetic oxides can be taken in the simpler form (5). The question has not answered definitely as yet.

\subsection{Specific features of the real-space pairing}

The operators $B_{i j}^{\dagger} B_{i j}$ and $A_{i m}^{\dagger} A_{i m}$ have only a vague relation to the particle-number operator $a_{i \sigma}^{\dagger} a_{j \bar{\sigma}}^{\dagger} a_{j \bar{\sigma}} a_{i \sigma}=n_{i \sigma} n_{j \bar{\sigma}}$, as the latter represents intersite (for $i \neq j$ ) particle-particle correlation function. However, the pairing part of the total energy diminishes if the average $\left\langle B_{i j}^{\dagger} B_{i j}\right\rangle$ is nonzero. This average has a nontrivial structure, namely $B_{i i}^{\dagger} B_{i i} \equiv 0$. The last property follows from the circumstance that the double occupancies are projected out on each site. This leads in turn, to a nontrivial structure of the superconducting gap. Both these properties follow from fact that the pairing operators are composed of the projected fermion operators $b_{i \sigma}^{\dagger} \equiv a_{i \sigma}\left(1-n_{i \bar{\sigma}}\right)$ and $b_{i \sigma} \equiv a_{i \sigma}\left(1-n_{i \bar{\sigma}}\right)$, and similarly for $f$ operators.

The second characteristic feature of the whole approach is that the projected Fermi operators have non-fermionic anticommutation relations. For example,

$$
\left\{b_{i \sigma}, b_{j \sigma}^{\dagger}\right\}=\left[\left(1-n_{i \bar{\sigma}}\right) \delta_{\sigma \sigma^{\prime}}-S_{i}^{\bar{\sigma}}\left(1-\delta_{\sigma \sigma^{\prime}}\right)\right] \delta_{i j} .
$$

Additionally, the hopping and the exchange-interaction terms do not commute with each others, so the motion of electrons and the spin-interaction (e.g. the spin-flip processes) are entangled with each other and result in a strongly correlated (non-Fermi liquid) metallic state. What is equally important, the kinetic-energy $\left(\sim t_{i j}\right)$ and the exchange $\left(\sim t_{i j}^{2} / U\right)$ can easily become comparable for filing close to the half filling $(n)$ and we can have a transformation form such metal to the localized antiferromagnetic state (or vice versa) or to the phase separation into antiferromagnetic 
islands immersed in the sea of $(1-n) N$ holes with ferromagnetic ordering of the spin inside the sea. The second state is rather unstable as the Coulomb repulsion introduced by the hole condensation in part of the system will charge the system and destabilize such phase-representation state. Nonetheless, antiferromagnetic state may coexist with the paired state. Such possibility arises from the identity which for the single narrow-band case reads

$$
\mathbf{S}_{i} \cdot \mathbf{S}_{j}-\frac{1}{4} \nu_{i} \nu_{j} \equiv-B_{i j}^{\dagger} B_{i j}
$$

and hence, the nonzero averages of the type $\left\langle B_{i j}\right\rangle$ may imply the nontrivial averages of $\left\langle S_{i}^{z}\right\rangle$ and vice versa. Likewise, from the analogous identity for the $f-c$ system, i.e.

$$
\mathbf{S}_{i} \cdot \mathbf{s}_{m}-\frac{1}{4} \nu_{i} n_{m} \equiv-A_{i m}^{\dagger} A_{i m}
$$

It follows, that the nontrivial structure of magnetic correlations expressed via $\left\langle\mathbf{S}_{i}\right\rangle$ and $\left\langle\mathbf{s}_{m}\right\rangle$ may implicate nontrivial structure of the averages $\left\langle A_{i m}^{\dagger}\right\rangle$ in some sort of mean field approximation discussed next. Obviously, there is a problem, to what extent the magnetic and the paired states can be regarded as different states in view of the identities (11) and (12). This question can be answered in a way empirically only when studding relative stability of different states obtained from an approximate treatment, as the exact solution of any of these models is not available.

\section{Renormalized mean-field solution: pure paired states}

We concentrate now on the analysis renormalized mean-field solution for both t-J model (5) and the Anderson-lattice with the Kondo-type pairing (9). The standard method of approach is either the Gutzwiller approximation (GA) or the slave-boson approach, the latter constructed in such a manner that it reduces to the Gutzwiller approximation in the simplest normal and paramagnetic state. However, in the case of magnetic and/or superconducting state, the SB approach introduces specific effective fields [25] which also renormalize the chemical potential. Recently, we have proposed a new method of approach [26] which combines the feature of the GA and SB methods in the mean-field approximation. We called it the statistically-consistent Gutzwiller approximation (SGA). We discuss this method on two examples of the models introduced above leaving the detailed elaboration of the method to separate publications.

\subsection{Extended t-J model}

Any theoretical approach to the paired state described by the Hamiltonian (6) must deal with two separate problems. First, it must deal with restrictions introduced with the double-occupancy exclusion (i.e. with the Gutzwiller projection) and second, it must decouple the pairing part into a tractable form (here in the mean-field form). The first problem is dealt with by introducing the Gutzwiller or related approximations. It relies on replacing the Gutwiller projected operators with the ordinary fermion operators with renormalized coefficients. In effect, the Hamiltonian (6) is transformed to the form

$$
\mathcal{H}_{t J}=\sum_{i j \sigma} t_{i j} g_{i j \sigma}^{t} a_{i \sigma}^{\dagger} a_{j \sigma}-\sum_{i j} J_{i j} g_{i j}^{J} A_{i j}^{\dagger} A_{i j}+(\text { three-site terms }),
$$

where $g_{\sigma}$ and $g^{J}$ are the renormalization factors, which restrict the motion of single particles $\left(g_{\sigma}^{t}\right)$ and bound singlet pair $\left(g^{J}\right)$, respectively, as the constraint on the double occupancies has been released. The explicit form of the renormalization factors can be obtained via different ways. The second step is to perform the Hartree-Fock type decoupling of the pairing part. For the sake of clarity we discuss next the simplest model with the pairing when only $\left(\mathbf{S}_{i} \cdot \mathbf{S}_{j}\right)$ part is taken only as the pairing part [11, 13] and then present the results for the full model.

The exchange part can be decoupled in the Hartree-Fock approximation and incorporates as nonzero a priori all bilinear averages obtained according to the prescription

$$
\hat{A} \hat{B} \simeq\langle\hat{A}\rangle \hat{B}+\langle\hat{B}\rangle \hat{A}-\langle\hat{A}\rangle\langle\hat{B}\rangle
$$


where $\langle\ldots\rangle$ denotes the statistical average. Employing this procedure, we obtain the truncated Hamiltonian (13) in the forms

$$
\begin{aligned}
\mathcal{H}_{t J}= & \sum_{i j \sigma} t_{i j} g_{i j}^{t} a_{i \sigma}^{\dagger} a_{j \sigma}-\mu \sum_{i \sigma} a_{i \sigma}^{\dagger} a_{j \sigma}-\frac{3}{4} \sum_{i j \sigma} J_{i j} g_{i j}^{J}\left(\chi_{j i} a_{i \sigma}^{\dagger} a_{j \sigma}+H . c .-\left|\chi_{i j}\right|^{2}\right) \\
& -\frac{3}{4} \sum_{i j \sigma} J_{i j} g_{i j}^{J}\left(\Delta_{i j} a_{j \sigma} a_{i \bar{\sigma}}^{\dagger}+H . c .-\left|\Delta_{i j}\right|^{2}\right),
\end{aligned}
$$

when $\chi_{i j} \equiv\left\langle a_{i \sigma}^{\dagger} a_{j \sigma}\right\rangle$ and $\Delta_{i j} \equiv\left\langle a_{i \bar{\sigma}} a_{j \sigma}\right\rangle$ are respectively the hoping amplitude and the so-called RVB gap parameter, both to be the determined self-consistently. The standard procedure encompasses diagonalizing the bilinear Hamiltonian (15) and solving subsequently the self-consistent (s-c) Bogoliubov-de Gennes (BdG) equations for $\chi_{i j}, \Delta_{i j}$ and $\mu$.

As has been noticed recently [13, 15], the solution based on the s-c BdG equations may not be entirely satisfactory. The reason for this is that the renormalization factors are complicated functions of the parameters. For example, a simple scheme extending GA [27] yields the following expressions for the spatially homogeneous case.

$$
\begin{aligned}
g_{i j}^{t} & =\frac{s x(1-x)}{\left(1-x^{2}\right)+4\left|\chi_{i j}\right|^{2}} \\
g_{i j}^{J} & =\frac{4(1-x)^{2}}{\left(1-x^{2}\right)^{2}+8 x^{2} \beta_{i j}^{-}(2)+16 \beta_{i j}^{\dagger}(4)}
\end{aligned}
$$

with $\beta_{i j}^{\dagger} \equiv\left|\Delta_{i j}\right|^{n} \pm\left|\chi_{i j}\right|^{n}$. The site $i, j$ are the neighboring sites $\langle i j\rangle$. For dependence as these above, the parameters $\Delta_{i j}$ and $\chi_{i j}$ determined variationally form the approximate form of the free-energy functional may not (and are not) equal to these determined self-consistently. Therefore, to achieve a consistency of the results from statistical -physics point of view, one introduces [13, 15] the constraints in the spirit of Bogoliubov $(-\mu N)$ term in the grand-canonical formalism in order to enforce the particle-number conservation. Explicitly, the effective Hamilltonian $H_{\lambda}$ with additional constraints providing the statistical consistency can be written up as [1]

$$
\begin{aligned}
\hat{H}_{\lambda} \equiv & \mathcal{H}_{t J}^{\mathrm{MFA}}-\sum_{i} \lambda_{i}^{(n)}\left(\sum_{\sigma} a_{i \sigma}^{\dagger} a_{i \sigma}-\left\langle n_{i}\right\rangle\right)-\sum_{\langle i j\rangle \sigma}\left[\lambda_{i j}^{\chi}\left(a_{i \sigma}^{\dagger} a_{j \sigma}-\chi_{i j}\right)+H . c .\right] \\
& -\sum_{\langle i j\rangle \sigma}\left[\lambda_{i j}^{\Delta}\left(a_{i \bar{\sigma}} a_{j \sigma}-\Delta_{i j}\right)+H . c .\right]
\end{aligned}
$$

where the Lagrange multipliers $\lambda_{1}^{(n)}, \lambda_{i j}^{\chi}$, and $\lambda_{i j}^{\Delta}$ play the role of molecular fields coupled to the corresponding averages. Hamiltonian (18) represents our starting Hamiltonian, which we will discuss for the spatially homogeneous state i.e. when $\left\langle n_{i}\right\rangle=n, \chi_{\langle i j\rangle}=\chi$ and $\Delta_{\langle i j\rangle}=\Delta$. In that situation $\lambda_{i}^{(n)}=\lambda, \lambda_{\langle i j\rangle}^{\chi}=\lambda^{\chi}$, and $\lambda_{\langle i j\rangle}^{\Delta}$.

The next step is the diagonalization of $H_{\lambda}$ via the Bogoliubov-Valatin canonical transformation, which yields

$$
H_{\lambda}=\sum_{\mathbf{k}} E_{\mathbf{k}}\left(\gamma_{\mathbf{k} 0}^{\dagger} \gamma_{\mathbf{k} 0}+\gamma_{\mathbf{k} 1}^{\dagger} \gamma_{\mathbf{k} 0}\right)+\sum_{\mathbf{k}}\left(\xi_{\mathbf{k}}-E_{\mathbf{k}}\right)+C,
$$

with quasiparticle energy $E_{\mathbf{k}}=\sqrt{\xi_{\mathbf{k}^{2}}+D_{\mathbf{k}}^{2}}$, the superconducting-gap parameter $D_{\mathbf{k}} \equiv$ $\sqrt{2} \sum_{\tau} D_{\tau} \cos k_{\tau}$, and the bare-band energy $\xi_{\mathbf{k}}=-2 \sum_{\tau} T_{\tau} \cos k_{\tau}-\mu-\lambda$. For a two dimensional system discussed in detail next $\tau=(x, y)$. Also, the renormalized hopping and gap parameters are, respectively

$$
T=-t+\frac{3}{4} J g^{J}+\lambda^{\chi}, D_{\tau}=\frac{3}{4} J g^{J} \Delta_{\tau}+\lambda^{\Delta} .
$$

We see that the parameters $t_{\langle i j\rangle}=-t$ and $D$ are influenced by the Lagrange multipliers $\left(\lambda^{\chi}, \lambda^{\Delta}\right)$; that is why they play an important role in the system dynamics. The remaining parameters are defined in the original paper [13]. 
Having defined the quasiparticle energies $E_{\mathbf{k}}$, we can construct the free energy functional the (and the free energy) for fermionic quasiparticles in a standard manner

$$
\mathcal{F}\left\{\chi, \Delta, \lambda^{\chi}, \lambda^{\Delta}, T\right\}=C+\sum_{\mathbf{k}}\left(\xi_{\mathbf{k}}-E_{\mathbf{k}}\right)-2 k_{\mathrm{B}} T \ln \left(1+\mathrm{e}^{-\beta E_{\mathbf{k}}}\right)
$$

which we minimize subsequently with respect to $\chi, \Delta, \mu, \lambda, \lambda^{\chi}$, and $\lambda^{\Delta}$ for a two dimensional square lattice and for the $\mathrm{d}$-wave symmetry of the superconducting gap $\Delta_{x}=-\Delta_{y}=\Delta$ then, $\lambda_{x}^{\Delta}=-\lambda_{y}^{\Delta}=\lambda^{\Delta}$. The results obtained variationally within the present, more complete method, differ from those obtained from the self-consistent procedure, as has been shown in detail in [13, 15]. In those papers it has been shown also explicitly, that it is possible to have a spontaneously broken translation symmetry with $\chi_{x} \neq \chi_{y}$ (the Pomeranchuk instability), which is induced by the electronic correlations.

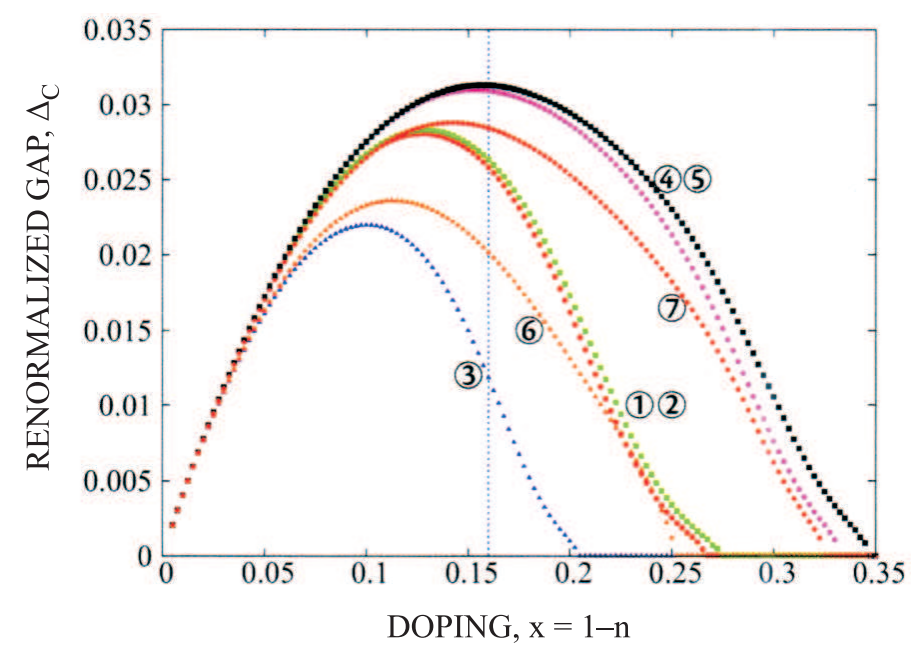

Figure 4. Doping dependence of the renormalized superconducting order parameter $\Delta_{\mathrm{c}}$. The curves 1-7 are explained in detail in [15] and correspond to various methods of solving Hamiltonian (2). The most important feature of the solution is the appearance of the upper critical concentration for the d-wave superconductivity disappearance. The vertical line defines roughly the optical doping, as in figure 1.

Instead of discussing in detail the above simplest situation with the statistical consistency conditions, we would like to mention one very important new undertaking in our group which we regard as an essentially important for all the available approaches. Namely, a new approach extending GA or SB methods has been recently proposed by Fukushima 28]. Implementation of this method to the t-J model leads to few very important novel properties within thus obtained renormalized mean-field theory. First of them is shown in figure 4 , where we present the doping $x \equiv 1-n$ dependence of the renormalized superconducting gap. The important feature of this dependence is the appearance of the upper critical concentration for the superconductivity disappearance irrespectively of what type of approximation we make as shown on the curves 1-6. We have induced there the full Hamiltonian (5), with reasonable values of the parameters: $J /|t|=0.3$ the ratio of the first two hopping parameters $t^{\prime} / t=-0.27$ (cf. curve 7) (for detailed explanation of the curves 1-6, see $[15]$ ). The values of the upper critical concentration is quite reasonable, when compared with experiment (cf. figure 1). Let us mention also, that the new method provides also reasonable value of the Fermi velocity and of the value of superconducting gap, both as a function of doping, at least in the overdoped regime, where the system is represented by an unconventional (almost-localized) Fermi liquid, with quasiparticle energies also adjusted variationally in the superconducting state. 


\subsection{Kondo-interaction induced pairing: spin-dependent effective masses and Fulde-Ferrell- Larkin-Ovchinnikov (FFLO) phase}

In this section we concentrate on a novel state discovered in strongly correlated system, namely on the Fulde-Ferrell-Larkin-Ovchinnikov state proposed theoretically many years ago [29].The motivation for us originating this work was the discovery [30] that this state has been observed in the heavy-fermion system CeCoIns 5 [30]. The interesting thing is that in the same system the spin-dependent masses have been observed earlier [31]. This last discovery was predicted earlier theoretically [32] and leads to rather strong Zeeman spin splitting of the band states. Therefore, it seemed natural to combine the two effects appearing simultaneously when the electronic correlations are included. However, one has to include then also the appearance of the effective field driven by the correlations and the real space pairing introduced in section 2.2 when the correlations are strong. All of these factors have been included the recent series of papers for both electron-gas [12, 14] and two-dimensional-band systems [33]. The results are overviewed briefly below.

\section{Spin-dependent masses}

For a quasiparticle gas of correlated particles, their energy in the applied field $h=g \mu_{\mathrm{B}} H_{\mathrm{a}}$, when the Landau quantization is neglected, is expressed as

$$
\xi_{\mathbf{k} \sigma}=\frac{\hbar^{2} k^{2}}{2 m \sigma}-\sigma h-\mu-\sigma h_{\mathrm{corr}}
$$

where $h_{\text {corr }}$ is the field induced by the correlations and spin-dependent mass enhancement $m_{\sigma} / m_{\mathrm{B}}$ is of the form in the limit of $U \rightarrow \infty$ for a single narrow band [1]

$$
\frac{m_{\sigma}}{m_{\mathrm{B}}}=\frac{1-n_{\sigma}}{1-n}=\frac{1-n / 2}{1-n}-\sigma \frac{\bar{m}}{2(1-n)} \equiv \frac{1}{m_{\mathrm{B}}}\left(m_{\mathrm{av}}-\frac{\sigma}{2} \Delta m\right),
$$

where $m_{\mathrm{B}}$ is the bare (band) mass, $\bar{m}=n_{\uparrow}-n_{\downarrow}$ is the system spin polarization, and $n$ is the band filling $\left(n=n_{\uparrow}+n_{\downarrow}\right)$. Also, $\Delta m=m_{\downarrow}-m_{\uparrow}$ is the mass difference and $m_{\mathrm{av}}=\left(m_{\uparrow}+m_{\downarrow}\right) / 2$ is the average quasiparticle mass. It is interesting to note that in the magnetic saturation limit we recover the band limit for the spin-majority subband, i.e. $m_{\uparrow} / m_{\mathrm{B}}=1$, whereas the heavy quasiparticles in the spin-minority band (with $m_{\downarrow} / m_{\mathrm{B}}=1 /(1-n)$ ) disappear. Two features are important here: (i) the masses are high in the almost-localized limit $(1-n) \ll 1$ and (ii) the mass $m_{\downarrow}$ in the spin-minority band is the heaviest, since due to the spin imbalance, those quasiparticles scatter very strongly (due to the presence of the large-magnitude Hubbard term $\sim U n_{i \uparrow} n_{i \downarrow}$ ). Additional features follows from the circumstance that we can "switch-off" completely the Hubbard interaction by applying the magnetic field and saturating magnetically the system. This is possible only (and is the case) because the field induced by the correlations enhances strongly the effect of applied magnetic field.

The brief analysis provided above delineates the principal message about what we mean by nonstandard quasiparticles in a (strongly) correlated system. First, they can become quite heavy, i.e. $m_{\mathrm{av}} / m_{\mathrm{B}} \gg 1$. Second they depend on the spin direction, what makes them distinguishable in the quantum-mechanical sense, since the mass in nonrelativistic quantum mechanics is an external (input) characteristic in the problem at hand. Third, the effective field driven by correlations can become very strong i.e. much stronger than any molecular field appearing in magnetism. All these microscopic properties must be determined self-consistently. This feature of nonstandard quasiparticles distinguishes the way they are introduced, as compared to that they are defined within the original phenomenological Landau- Fermi-liquid theory, where the enhancement of the effective mass and of the magnetic susceptibility is expressed in terms of interaction parameters, which are not determined within the scheme. Additionally, in the Landau scheme the enhancement factors are determined by including the interaction only among the quasiparticles at and/or in close vicinity of the Fermi surface. Here, all the particles mutually influence each other, what is expressed via an integration over all occupied states when solving appropriate self-consistent equations. 


\section{Nonstandard Cooper-pair states}

The effective-mass distinguishable quasiparticles will form also nonstandard Cooper pair 12 , 34]. This property is illustrated in figure 5, where the standard Cooper-pair and that with the spindependent masses are shown. The crucial consequence of the mass-inequivalence is demonstrated

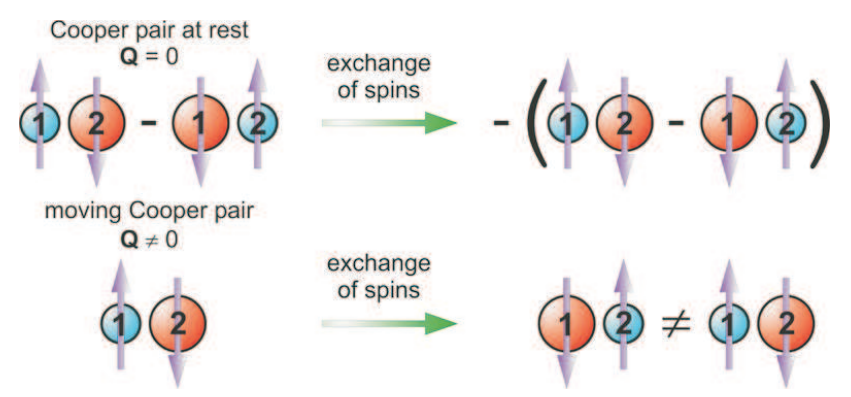

Figure 5. Behavior of the spin part of the Cooper-pair wave function under transposition of particles labeled as $1=\uparrow$ and $2=\downarrow$ at rest (upper row, antisymmetry preserved) and moving Cooper pair with center-of-mass momentum $\hbar \mathbf{Q} \neq 0$ (lower row, no definite symmetry).

explicitly in figure 6 , where the transition from Cooper pair at rest to the moving-pair state is observed at the critical field, that is also accompanied by the transition from the pure spin-singlet state to the spin-specific state $\chi_{\uparrow}(1) \chi_{\downarrow}(2)$. Further analysis of this point is carried out in detail elsewhere [12]. Note that the moving Cooper-pair state is a precursor of the FFLO-state appearance.

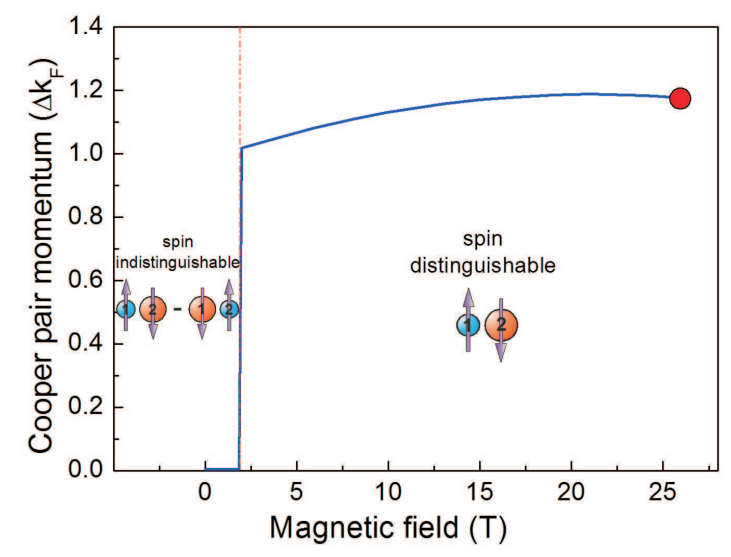

Figure 6. Optimal Cooper-pair center-of-mass momentum $\mathbf{Q}$ versus applied magnetic field. For high enough fields, $|\mathrm{Q}| \approx \Delta k_{\mathrm{F}}$, where $\Delta k_{\mathrm{F}}$ is the Fermi omentum difference for the two spin subbands. The nature of the spin part of the wave function in each regime is shown. The solid circle at the curve right marks the Pauli limiting critical field.

\section{Superconducting state: Fulde-Ferrell-Larkin-Ovchinnikov state}

We will describe next the Fulde-Ferrell-Larkin-Ovchinnikov (FFLO) state for the model heavyfermion system starting from Hamiltonian (9) with the hybrid pairing. This Hamiltonian in the simplest Gutzwiller approximation yields the following effective BCS-type Hamiltonian

$\mathcal{H}=\sum_{\mathbf{k} \sigma}\left[\epsilon_{\mathbf{k} \sigma} c_{\mathbf{k} \sigma}^{\dagger} c_{\mathbf{k} \sigma}+\tilde{\epsilon} f_{f \sigma} f_{\mathbf{k} \sigma}^{\dagger} f_{\mathbf{k} \sigma}+\tilde{V}_{\mathbf{k} \sigma} f_{\mathbf{k} \sigma}^{\dagger} c_{\mathbf{k} \sigma}+\tilde{V}_{\mathbf{k} \sigma}^{*} c_{\mathbf{k} \sigma}^{\dagger} f_{\mathbf{k} \sigma}\right]-\frac{2}{\epsilon+U} \frac{1}{N} \sum_{\mathbf{k k}^{\prime} Q} V_{\mathbf{k}} V_{\mathbf{k}^{\prime}}^{*} A_{\mathbf{k} \mathbf{Q}}^{\dagger} A_{\mathbf{k}^{\prime} \mathbf{Q}}$ 
with

$$
A_{\mathbf{k} \mathbf{Q}}=\frac{1}{\sqrt{2}}\left[f_{\mathbf{k}+\mathbf{Q} / \mathbf{2} \uparrow}^{\dagger} c_{-\mathbf{k}+\mathbf{Q} / \mathbf{2} \downarrow}^{\dagger}-f_{\mathbf{k}+\mathbf{Q} / \mathbf{2} \downarrow}^{\dagger} c_{-\mathbf{k}+\mathbf{Q} / \mathbf{2} \uparrow}^{\dagger}\right] .
$$

The single-particle part can be easily diagonalized by moving to the hybridized basis, whereas the pairing part is represented by a separable pairing potential. We proceed with the transformation to the hybridized basis first, which yields the following transformed pairing part among the quasiparticles in the lower hybridized band (appropriate for $\left\langle f_{i \sigma}^{\dagger} f_{i \sigma}\right\rangle+\left\langle c_{i \sigma}^{\dagger} c_{i \sigma}\right\rangle \leqslant 1$ )

$$
\begin{aligned}
\mathcal{H}= & \sum_{\mathbf{k} \sigma} E_{\mathbf{k} \sigma} \alpha_{\mathbf{k} \sigma}^{\dagger} \alpha_{\mathbf{k} \sigma}-\frac{4}{\epsilon_{\mathrm{f}}+U} \sum_{\mathbf{k} \mathbf{k}^{\prime} \mathbf{Q}} \frac{\left|V_{\mathbf{k}} V_{\mathbf{k}^{\prime}}\right|^{2}\left(q_{\sigma} q_{\bar{\sigma}}\right)^{1 / 2}}{\left[\left(\epsilon_{\mathbf{k} \sigma}-\tilde{\epsilon}_{f \sigma}\right)^{2}+\left|\tilde{V}_{\mathbf{k} \sigma}\right|^{2}\right]^{1 / 2}\left[\left(\epsilon_{\mathbf{k}^{\prime} \sigma}-\tilde{\epsilon}_{f \bar{\sigma}}\right)^{2}+\left|\tilde{V}_{\mathbf{k}^{\prime} \bar{\sigma}}\right|^{2}\right]^{1 / 2}} \\
& \times \alpha_{\mathbf{k}+\mathbf{Q} / 2 \uparrow}^{\dagger} \alpha_{-\mathbf{k}+\mathbf{Q} / 2 \downarrow}^{\dagger} \alpha_{-\mathbf{k}^{\prime}+\mathbf{Q} / 2 \downarrow} \alpha_{\mathbf{k}^{\prime}+\mathbf{Q} / 2 \uparrow},
\end{aligned}
$$

where the quasiparticle energy in the normal state is given by

$$
E_{\mathbf{k} \sigma} \equiv \frac{\epsilon_{\mathbf{k} \sigma}+\tilde{\epsilon}_{f \sigma}}{2}-\left[\left(\frac{\epsilon_{\mathbf{k} \sigma}+\tilde{\epsilon}_{f \sigma}}{2}\right)^{2}+\left|\tilde{V}_{\mathbf{k} \sigma}\right|^{2}\right]^{1 / 2}
$$

In all these equations $\epsilon_{\mathbf{k} \sigma}=\epsilon_{\mathbf{k}}-\sigma h-\mu, \epsilon_{\mathbf{k} \sigma}=\epsilon_{\mathrm{f}}-\sigma h-\mu, \tilde{V}_{\mathbf{k} \sigma}=V_{\mathbf{k}} q_{\sigma}^{1 / 2}$, where the normalization factor is takes in the Gutzwiller approximation [33]. For the constant (intraatomic) form of the hybridization $V_{\mathbf{k}}=V=-|V|$, we obtain effectively single-band model discussed in detail in [34], with heavy electrons of primary $f$ type, i.e $\alpha_{\mathbf{k} \sigma} \simeq f_{\mathbf{k} \sigma}$ if $1-n \ll 1$. Below we present only the principal features of the phase diagram leaving the details for the full papers [12, 14, 33]. Note that in general we have here the center-of-mass momentum of the Cooper pair $\mathbf{Q} \neq 0$ to include a possibility of the FFLO phase appearance (strictly speaking, we consider only the Fulde-Ferrell case).

In the standard Hartree-Fock approximation with anomalous averages, Hamiltonian (26) in the narrow band limit reduces to the form

$$
\begin{aligned}
\mathcal{H}= & \sum_{\mathbf{k} \sigma}\left(\epsilon_{\mathbf{k} \sigma}-\mu\right) f_{\mathbf{k} \sigma}^{\dagger} f_{\mathbf{k} \sigma}-g \mu_{\mathrm{B}} H \sum_{\mathbf{k}}\left(f_{\mathbf{k} \uparrow}^{\dagger} f_{\mathbf{k} \uparrow}-f_{\mathbf{k} \downarrow}^{\dagger} f_{\mathbf{k} \downarrow}\right) \\
& +\sum_{\mathbf{k}}\left(\Delta_{\mathbf{k} \mathbf{Q}}^{*} f_{\mathbf{k} \uparrow} f_{-\mathbf{k}+\mathbf{Q} \downarrow}+H . c .\right)+N \frac{\left|\Delta_{\mathbf{k} \mathbf{Q}}\right|^{2}}{V_{0}}
\end{aligned}
$$

with the single-particle energy parameterized in the hight-binding approximation, which in for the case of square lattice takes the form

$$
\epsilon_{\mathbf{k} \sigma} \equiv q_{\sigma}\left[-2 t\left(\cos k_{x}+\cos k_{y}\right)+4 t^{\prime} \cos k_{x} \cos k_{y}\right]
$$

and $q_{\sigma}$ is, as before, the Gutwiller band narrowing factor (the spin-dependent mass enhancement) and the superconducting gap is determined from the self-consistent equation

$$
\Delta_{\mathbf{k} \mathbf{Q}}=-\frac{V_{0}}{N} \sum_{\mathbf{k}^{\prime}} \gamma_{\mathbf{k}} \gamma_{\mathbf{k}^{\prime}}\left\langle f_{-\mathbf{k}^{\prime}+\mathbf{Q} \downarrow} f_{\mathbf{k}^{\prime} \uparrow}\right\rangle .
$$

Additionally, $V_{0}$ is the pairing magnitude which for the constant hybridization has the form

$$
V_{0}=-\frac{4 V^{2}\left(q_{\sigma} q_{\bar{\sigma}}\right)^{1 / 2}}{\epsilon_{\mathrm{f}}+U}
$$

and the factors $\gamma_{\mathbf{k}}$ and $\gamma_{\mathbf{k}^{\prime}}$ correspond to the separable $\mathbf{k}$-dependent factors in (26) divided by $V^{2}$.

Next, we carry out the approximate form of the Bogoliubov transformation which in the meantime acquired the name of the Bogoliubov-Valatin-de Gennes-Nambu transformation! For that 
purpose we represent (28) in the matrix form

$$
\begin{aligned}
\mathcal{H}= & \sum_{\mathbf{k}}\left(f_{\mathbf{k} \uparrow}^{\dagger}, f_{-\mathbf{k}+\mathbf{Q} \downarrow}\right)\left(\begin{array}{cc}
\epsilon_{\mathbf{k} \uparrow}-g \mu_{\mathrm{B}} H-\mu & \Delta_{\mathbf{k}, \mathbf{Q}} \\
\Delta_{\mathbf{k}, \mathbf{Q}}^{*} & -\epsilon_{-\mathbf{k}+\mathbf{Q} \downarrow}-g \mu_{\mathrm{B}} H+\mu
\end{array}\right)\left(\begin{array}{c}
f_{\mathbf{k} \uparrow} \\
f_{-\mathbf{k}+\mathbf{Q} \downarrow}^{\dagger}
\end{array}\right) \\
& +\sum_{\mathbf{k}}\left(\epsilon_{\mathbf{k} \downarrow}+g \mu_{\mathrm{B}} H-\mu\right)+N \frac{\Delta_{\mathbf{Q}}^{2}}{V_{0}} .
\end{aligned}
$$

The transformation to the quasiparticle representation has the usual form

$$
\left(\begin{array}{c}
\tilde{\alpha}_{\mathbf{k}} \\
\tilde{\beta}_{\mathbf{k}}^{\dagger}
\end{array}\right)=\left(\begin{array}{cc}
u_{\mathbf{k}} & v_{\mathbf{k}} \\
-v_{\mathbf{k}} & u_{\mathbf{k}}
\end{array}\right)\left(\begin{array}{c}
f_{\mathbf{k} \uparrow} \\
f_{-\mathbf{k}+\mathbf{Q} \downarrow}^{\dagger}
\end{array}\right)
$$

with the Bogoliubov coherence factors given now by the relations

$$
\begin{aligned}
& u_{\mathbf{k}}=\left[\frac{1}{2}\left(1+\frac{\epsilon_{\mathbf{k} \uparrow}+\epsilon_{-\mathbf{k}+\mathbf{Q} \downarrow}-2 \mu}{\sqrt{\left(\epsilon_{\mathbf{k} \uparrow}+\epsilon_{-\mathbf{k}+\mathbf{Q} \downarrow}-2 \mu\right)^{2}+4 \Delta_{\mathbf{k Q}}^{2}}}\right)\right]^{1 / 2}, \\
& v_{\mathbf{k}}=\left[\frac{1}{2}\left(1-\frac{\epsilon_{\mathbf{k} \uparrow}+\epsilon_{-\mathbf{k}+\mathbf{Q} \downarrow}-2 \mu}{\sqrt{\left(\epsilon_{\mathbf{k} \uparrow}+\epsilon_{-\mathbf{k}+\mathbf{Q} \downarrow}-2 \mu\right)^{2}+4 \Delta_{\mathbf{k Q}}^{2}}}\right)\right]^{1 / 2} .
\end{aligned}
$$

The quasiparticle energies in the phase with $\mathbf{Q} \neq 0$ are

$$
E_{\mathbf{k} \mathbf{Q} \alpha}=\frac{1}{2}\left(\epsilon_{\mathbf{k} \uparrow}-\epsilon_{-\mathbf{k}+\mathbf{Q} \downarrow}\right)-g \mu_{\mathrm{B}} H \alpha \frac{1}{2}\left[\left(\epsilon_{\mathbf{k} \uparrow}+\epsilon_{-\mathbf{k}+\mathbf{Q} \downarrow}-2 \mu\right)^{2} 4\left|\Delta_{\mathbf{k} \mathbf{Q}}\right|^{2}\right]^{1 / 2},
$$

where sign factor $\alpha= \pm$ corresponds to the electron or hole excitations, respectively.

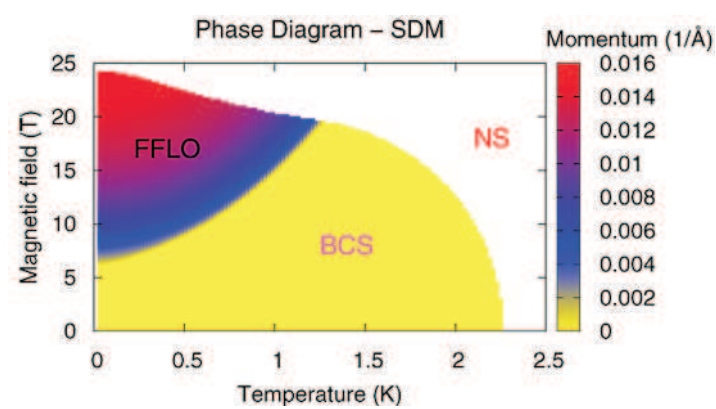

(a)

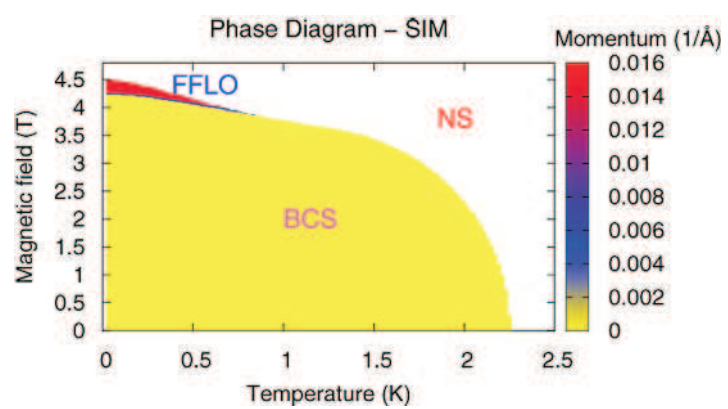

(b)

Figure 7. Phase diagram for the cases with the spin-dependent (a) and the spin-independent masses (b). Light region corresponds to $\mathbf{Q}=0$ (BCS phase), the darker one to $\mathbf{Q} \neq 0$ (FFLO phase) and the white to normal state. Note that with increasing temperature, the transition from BCS to FFLO state occurs at higher fields, tin qualitative agreement with experimental results [30]. The FFLO phase is stable in an extended $H_{\mathrm{a}}-T$ regime only in the SDM case.

Having discussed the explicit expression for the fermionic quasiparticle excitations, we can construct the free energy functional, as well as determine the system of self-consistent equations for $\Delta_{\mathbf{k Q}}, \mu$, and $\bar{m}$; we also optimize the energy with respect to the magnitude of the wave vector $\mathbf{Q}$. One should also mention that for the electron-gas situation we have included explicitly in the effective Hamiltonian the correlation field $h_{\text {corr }}$, which we optimize, whereas for the two-dimensional band structure (29) we have been able so far to carry out only the whole analysis in the Gutzwiller approximation for the Hamiltonian (24), before the pairing is included. In figures 7 and 8 we provide the exemplary phase diagrams on the plane temperature $T$ - applied magnetic field $H$ 


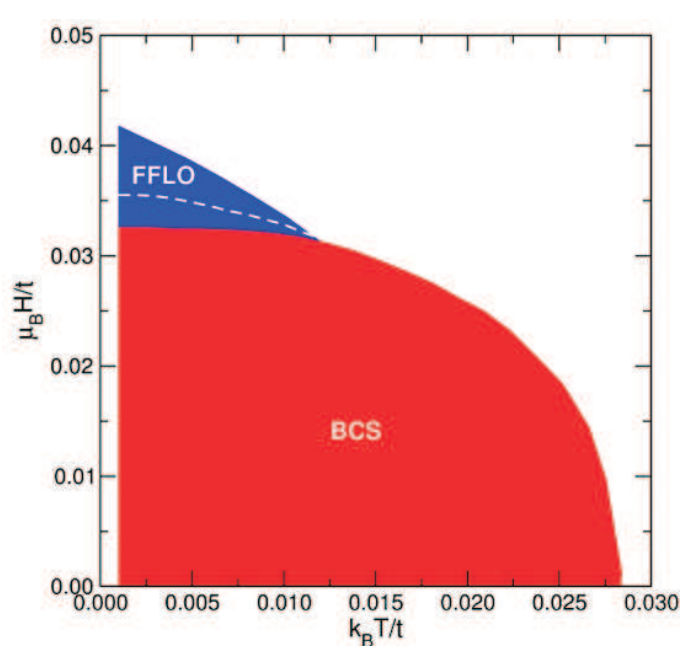

(a)

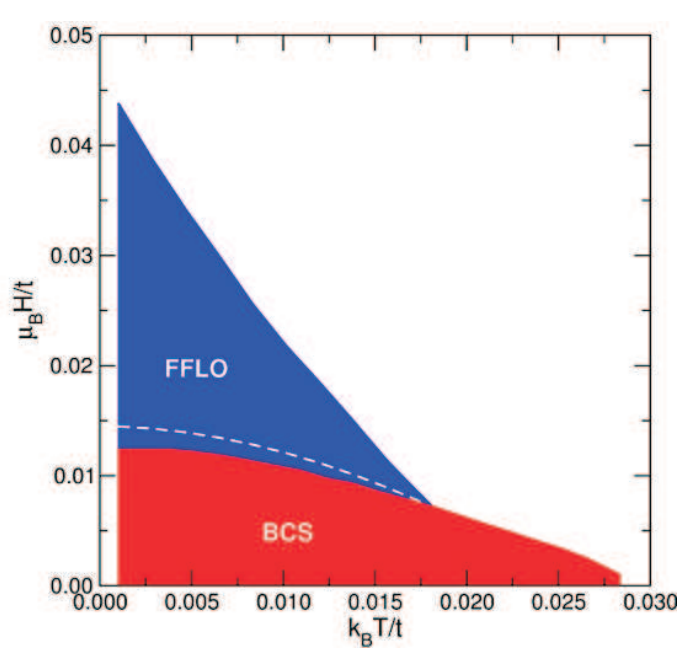

(b)

Figure 8. Phase boundaries for a two-dimensional $d$-wave superconductor with both the spinindependent masses (SIM) (a) and with the spin-dependent masses (SDM) (b). The FFLOBCS transition line is of discontinuous nature. The dashed line marks the stability limit of the BCS state as determined by the value of the second critical field $H_{\mathrm{c} 2}$ for the BCS state. The values of parameters are $n=0.97$ and $V_{0}=12.5 \mathrm{~K}$. For these values of the parameters, the superconducting transition temperature is $T=2.5 \mathrm{~K}$ and the uppermost critical field for the FFLO phase is above $6 T$. Note that the FFLO state is robust in the situation with SDM and this result is one of the principal features of the present discussion.

for the three-dimensional gas [12] and square-lattice cases [33], respectively. The most important feature coming out of these figures are: (i) the BCS state (i.e. that with $\mathbf{Q}=0$ ) is quite robust in the lower fields and higher temperatures, (ii) the inclusion of the effective-mass spin dependence leads to a remarkable extension of regime of the FFLO stability, (iii) in the FFLO phase the upper critical field of the transition to the normal phase is much higher than that for the BCS superconducting state, and (iv) the first-order BCS $\rightarrow$ FFLO transition can be accompanied by a weak metamagnetic transition [12]. One should also not that the detailed analysis of the FFLO state is carried out elsewhere [33]. Also, the full SGA analysis of the superconducting states within the full statistically-consistent Gutzwiller approach (SGA) for the present model is still to be finalized.

\section{Concluding remarks}

In this brief review I have tried to demonstrate the usefulness of the concept of an unconventional Fermi liquid for such diverse phenomena as high temperature superconductivity (as represented by t-J model) and heavy-fermion superconductivity (represented by the Kondo pairing) and particularly, the Fulde-Ferrell-Larkin-Ovchinnikov-state stability in the latter case (on the example of narrow-band limit with the Kondo-interaction induced pairing). The statisticallyconsistent (SGA) method proposed by us [26] is probably the most general Fermi-liquid approach possible for almost localized correlated systems, as we renormalize all relevant quantities: introduce the spin-dependent mass renormalization, as well as the effective field and the chemical-potential shift, all induced by the interparticle correlations. In addition to that, specific purely electronic mechanism of pairing in real space, leads to the BCS-type of Hamiltonian for all electrons and with a nontrivial (d-wave) form of the superconducting gap. Inclusion of quantum fluctuations in the mean-field SGA solution is possible [26], but first one has to implement the SGA method completely by incorporating in it also the possibility of coexistence of superconductivity with antiferromagnetism. Also, the role of the intersite Coulomb (for the t-J model) and the interorbital 
Coulomb (for the model with Kondo pairing) has to clarified, as it works against any of the intersite real-space pairing driven by the electronic correlations. We should be able to see a progress along these lines in the near future.

\section{Acknowledgement}

The work was supported by Grant No. N N202 128736 from our Ministry of Science and Higher Education. The results reviewed in this paper are based largely on the detailed work of my Ph.D. students Jakub Jȩdrak and Jan Kaczmarczyk. Numerous discussions with Profs. Maciej Maśka and Marcin Mierzejewski are appreciated. Technical help of Dr. Danuta Goc-Jagło is also acknowledged.

\section{References}

1. Bogoliubov N.N., Nuovo Cimento, 1958, 7, 794;Bogoliubov N.N., Tolmachev V.V., Shirkov D.V., A New Method in the Theory of Superconductivity, english transl. Consultant Bureau, New York, 1959: Fortschritte der Physik, 1958, 6, 605.

2. Valatin J.G., Nuovo Cimento, 1958, 7, 843.

3. de Gennes P.G., Superconductivity of Metals and Alloys, chapter 5. Addison-Wesley Publ., Reading, 1989.

4. Morel P., Anderson P.W., Phys. Rev., 1962, 125, 1263.

5. Keimer B.N., Belk N. , Birgeneau R.J., Cassanho A., Chen C.Y., Greven M., Kastner M.A., Aharony A., Endoh Y., Erwin R.W., Shirane G., Phys. Rev. B, 1992, 46, 14034; Damascelli A., Hussin Z., Shen Z.-X., Rev. Mod. Phys., 2003, 75, 473.

6. Kastner M.A., Birgeneau R.J., Shirane G., Endoh Y., Rev. Mod. Phys., 1998, 70, 897.

7. Ruckenstein A.E., Hirschfeld P.J., Appel J., Phys. Rev. B, 1987, 36, 857.

8. Spałek J., Phys. Rev. B, 1988, 37, 533.

9. Zhang F.C., Rice T.M., Phys. Rev. B, 1988, 37, 3759.

10. Anderson P.W. - In: Frontiers and Borderlines in Many-Particle Physics, edited by Broglia R.A., Schrieffer J.R. North Holland, Amsterdam, 1988, p. 1ff.

11. Lee P.A., Nagaosa N., Wen X.-G., Rev. Mod. Phys., 2008, 78, 17; Ogata M., Fukuyama H., Rep. Prog. Phys., 2008, 71, 036501.

12. Kaczmarczyk J., Spałek J., Phys. Rev. B, 2009, 79, 214519.

13. Jędrak J., Spałek J., Phys. Rev. B, 2010, 81, 073108.

14. Kaczmarczyk J., Spałek J., J. Phys.: Condens. Mat., 2010, 22, 355702.

15. Jȩdrak J., Spałek J. Preprint arXiv:1004.4282, submitted to Phys. Rev. B.

16. Edegger B., Muthukumar V.N., Gross C., Adv. Phys., 2007, 56, 927.

17. Anderson P.W., Phys. Rev., 1959, 115, 2; Solid State Physics, edited by Seitz F., Turnbull D. Academic Press, New York, 1963, p. 99-214.

18. Zaanen J., Sawatzky G.A., Allen J.W., Phys. Rev. Lett., 1985, 55, 418; Zaanen J., Sawatzky G.A., Allen J.W., J. Magn. Magn. Mat., 1986, 54-57, 607; Zaanen J., Sawatzky G.A., J. Solid State Chem., $1990, \mathbf{8 8}, 8$.

19. Spałek J., Oleś A.M. Preprint TPJU 6/76 (1976); Spałek J., Oleś A.M., Physica B, 1977, 86-88, 375; Chao K.A., Spałek J., Oleś A.M., J. Phys. C, 1977, 10, L271; Chao K.A., Spałek J., Oleś A.M., Phys. Rev. B, 1978, 18, 3453.

20. Spałek J., Acta Phys. Polon. A, 2007, 111, 409.

21. Spałek J., Condens. Matter Phys., 2008, 11, 455.

22. Klejnberg A. Ph. D. Thesis, Jagiellonian University, Kraków, 2006 (unpublished).

23. Schrieffer J.R., Wolff P.A., Phys. Rev., 1966, 149, 491; Spałek J., Oleś A.M., Chao K.A., Phys. Rev. B, 1978, 18, 3748 .

24. Spałek J., Phys. Rev. B, 1988, 38, 208.

25. Spałek J., Wójcik W. - In: Spectroscopy of Mott Insulators and Correlated Metals, edited by Fujimori A., Tokura Y. Springer, Berlin, 1994, p. 49-65.

26. Jȩdrak J., Kaczmarczyk J., Spałek J. Preprint arXiv:1008.0021, submitted to Phys. Rev. B.

27. Raczkowski M., Acta Phys. Polon. A, 2008, 114, 107.

28. Fukushima N., Phys. Rev. B, 2008, 78, 115105.

29. Fulde P., Ferrell R.A., Phys. Rev., 1964, 135, A550; Larkin A.I., Ovchinnikov Yu.N., Zh. Exp. Teor. Fiz., 1964, 47, 1136; Larkin A.I., Ovchinnikov Yu.N., Sov. Phys. JETP, 1965, 20, 762. 
30. McCollam A., Julian S.R., Rourke P.M.C., Aoki D., Flouquet J., Phys. Rev. Lett., 2005, 94, 186401.

31. Kumagai K., Saitoh M., Oyaizu T., Furukawa Y., Takashima S., Nohara M., Takagi H., Matsuda Y., Phys. Rev. Lett., 2006, 97, 227002; Miclea C.F., Nicklas M., Parker D., Maki K., Sarrao J.L., Thompson J.D., Sparn G., Steglich F., Phys. Rev. Lett., 2006, 96, 117001; Kakuyanagi K., Saitoh M., Kumagai K., Takashima S., Nohara M., Takagi H., Matsuda Y., Phys. Rev. Lett., 2005, 94, 047602.

32. Spałek J., Gopalan P., Phys. Rev. Lett., 1990, 64, 2823; Korbel P., Spałek J., Wójcik W., Acquarone M., Phys. Rev. B, 1995, 52, R2213; Spałek J., Phys. Stat. Sol. (b), 2006, 243, 78 (Editor's Choice).

33. Maśka M., Mierzejewski M., Kaczmarczyk J., Spałek J., Phys. Rev. B, 2010, 82, 054509.

34. Wróbel P., Starypan Z., Kaczmarczyk J., Spałek J., Acta Phys. Polon. A, 2006, 109, 541.

\title{
Надпровідні стани у сильно скорельованих системах 3 нестандартними квазічастинками і спарюванням у реальному просторі: границя нетрадиційної Фермі-рідини
}

\author{
Й. Спалек 112 \\ 1 Інститут фізики ім. Мар'яна Смолуховського, Ягелонський університет, Краків, Польща \\ 2 Факультет фізики і прикладних комп'ютерних наук, AGH університет науки і технології, \\ Краків, Польща
}

\begin{abstract}
Ми використовуємо концепцію узагальненої (майже локалізованої) Фермі рідини, що складається 3 нестандартних квазічастинок із спін залежними ефективними масами і ефективним полем, індукованим електронними кореляціями. Фермі рідина отримана в рамках так званого статистичноузгодженого наближення Гутцвіллера (SGA), що було недавно запропоноване [cf. J. Jędrak et al., arXiv: 1008.0021] і описує електронні стани скорельованої квантової рідини. Особливий наголос робиться на спарюванні у реальному просторі, яке викликане електронними кореляціями, стані Фульде-Феррелла важкої ферміонної рідини і d-хвильовому надпровідному стані високотемпературних мідних надпровідників у випадку перелегування. Обговорюється відповідна фазова діаграма, яка демонструє, зокрема, границі стійкості станів типу Бардіна-Купера-Шріффера.
\end{abstract}

Ключові слова: скоорельовані ферміони, високотемпературна надпровідність, нетрадиційна надпровідність, спарювання в реальному просторі, важкі ферміони, модель $t$-J, спарювання Кондо 